
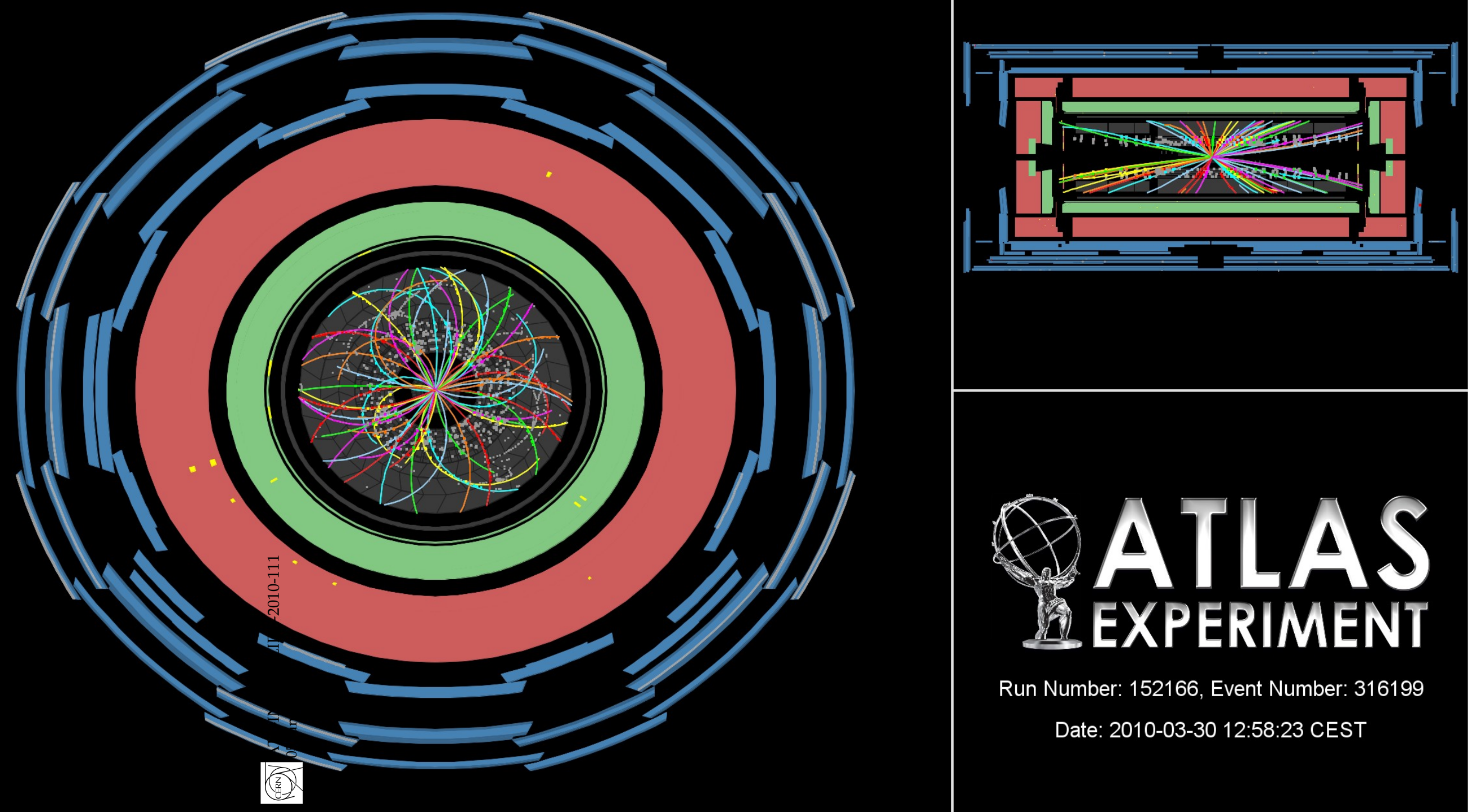

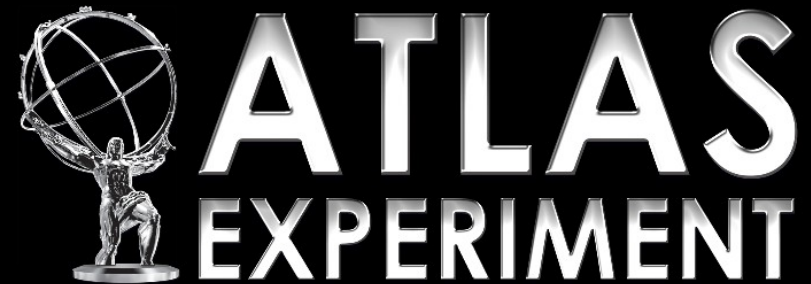

Run Number: 152166, Event Number: 316199 Date: $2010-03-30$ 12:58:23 CEST
Alignment of ATLAS Inner Detector Tracking System
Shih-Chieh Hsu on behalf of ATLAS Lawrence Berkeley National Lab June 82010 IPRD10 in Siena Italy 


\section{A Toroidal LHC Apparatus}

Outline

- Introduction

- Methodology focus on track based alignment

- Strategy

- Results (2009 2010)

- Validation

- Summary

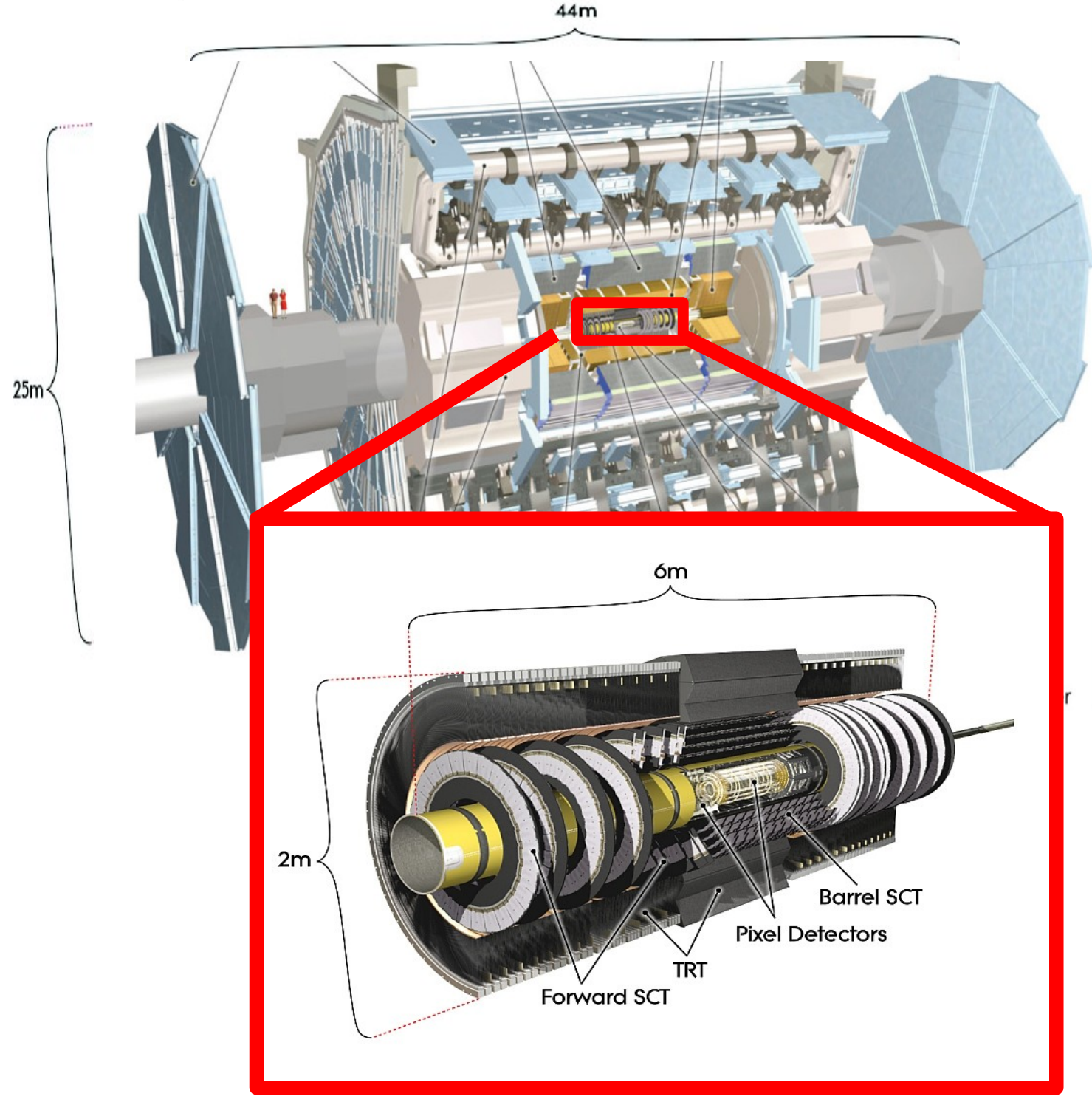



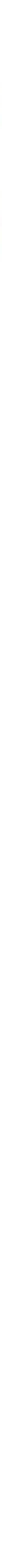

- Inner Detector Specification

- transverse momentum resolution required to be less than $30 \%$ at $500 \mathrm{GeV} \mathrm{p}_{\mathrm{T}}$ $\sigma\left(\mathrm{p}_{\mathrm{T}}\right) / \mathrm{p}_{\mathrm{T}}=0.05 \% \mathrm{p}_{\mathrm{T}}[\mathrm{GeV}] \oplus 1 \%$

- Good b-tagging performance $(50 \%$ b-jet efficiency with light-jet rejection factor 1000 for top analysis) Impact parameter resolution $(0.25<|\eta|<0.5)$ $\sigma\left(\mathrm{d}_{0}\right)=10 \mu \mathrm{m} \oplus 140 \mu \mathrm{m} / \mathrm{p}_{\mathrm{T}}[\mathrm{GeV}]$

- Alignment Challenges

- More than 35000 degree of freedom PIX: 1744 SCT: 4088 TRT: 176

- Different scale of resolution PIX: $\mathrm{O}(10 \mu \mathrm{m})$ SCT: $\mathrm{O}(30 \mu \mathrm{m})$

TRT: $\mathrm{O}(140 \mu \mathrm{m})$ 


\section{irrerm \\ Methodology \& Algorithms}

- Hardware: Assembly/Survey Measurements external measurement during installation

- Hardware: Interferometry laser interference monitors of SCT structure in real time

- Software:Track Based Alignment (Focus of this talk. All methods are iterative)

- Global $\chi^{2}$ correlate all modules along the same track to construct alignment with full systems

- Local $\chi^{2}$ correlate modules through iterations to solve alignment constants with smaller system

- Robust Alignment

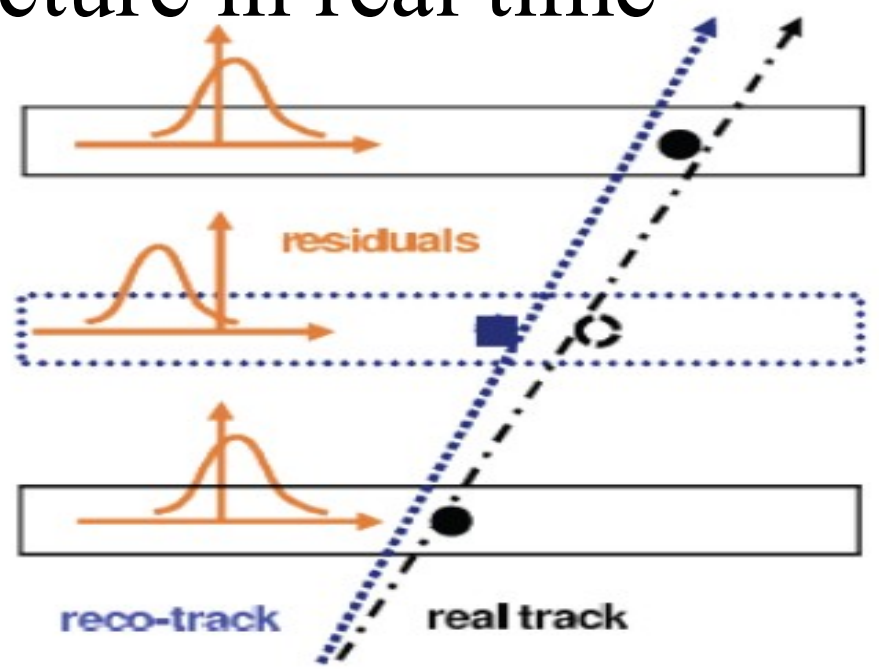
use residual (equivalent to constant hit errors) and overlap residuals

$$
\begin{aligned}
& \chi^{2}=\sum_{\text {hits } \mathrm{i}}\left(\frac{m_{i}-h_{i}(x)}{\sigma_{i}}\right)^{2} \\
& \Delta \alpha \equiv \alpha-\alpha_{0}=-\left(\left.\frac{d^{2} \chi^{2}}{d \alpha^{2}}\right|_{\alpha_{0}}\right)^{-1} \frac{d \chi^{2}\left(\alpha_{0}\right)}{d \alpha}
\end{aligned}
$$




\section{Computing Model}

- Dedicated ID calibration stream for alignment

- Successfully test each computing element in the GRID May 2010

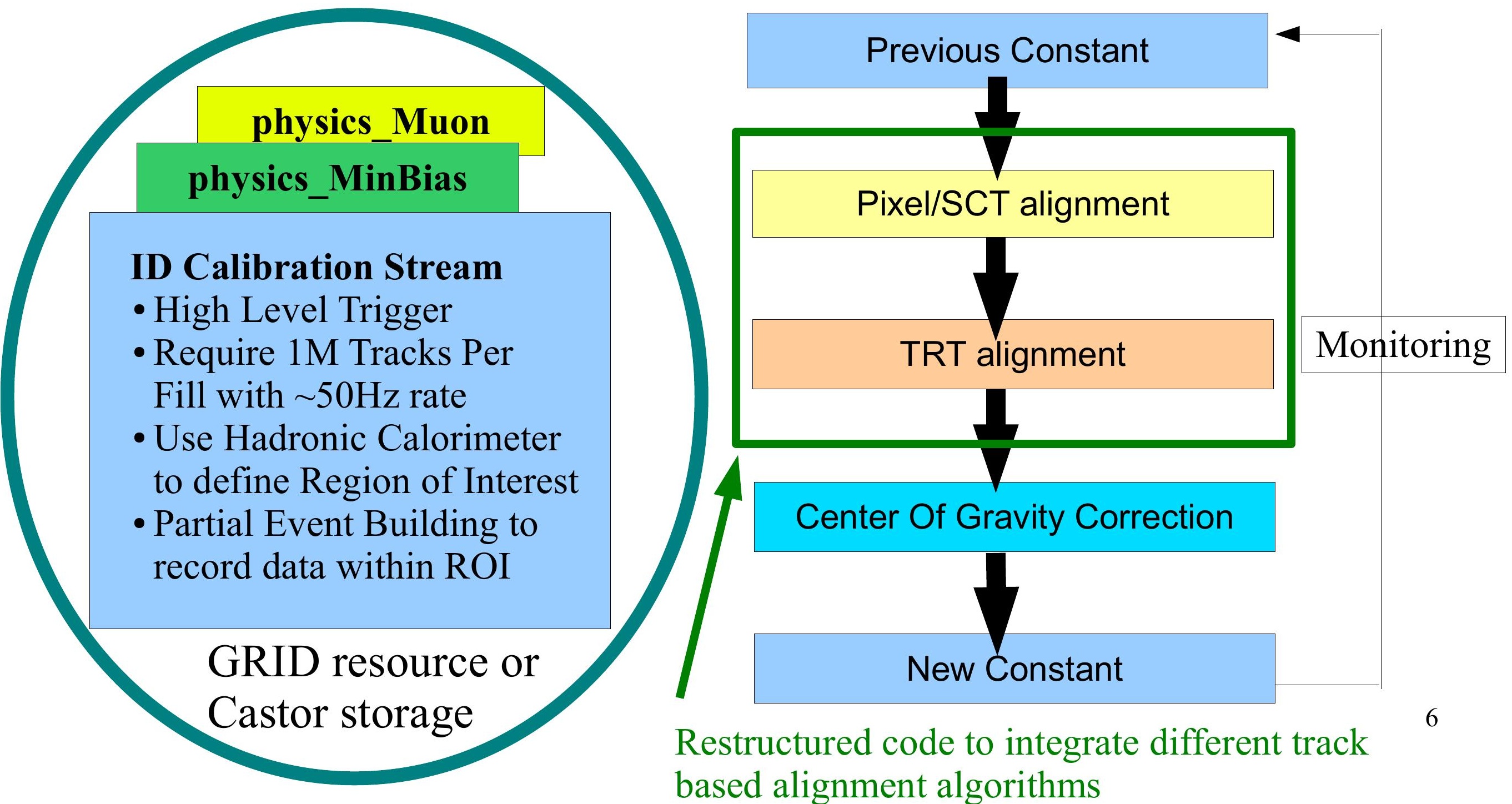




\section{Alignment Strategy}

\section{8 \\ - Commissioning Phase}

- Broaden hit errors to guarantee hit efficiency; Start from nominal

- Cosmic alignment with non-uniform illumination rate. Endcap has poor

Dec $\sim$

\section{0} March

\section{- Operation Phase}

- Start from commission alignment. Use precision errors. Add beam spot constraint to pursue better alignment

- Routinely monitor alignment quality by using dedicated processing stream and Grid resource
Dedicate TRT Trigger to record ID tracks efficiently for alignment

High statistics Calorimeter Cosmics Trigger to record events in parallel with stable beams

Dedicate ID Calibration stream to record isolated high $\mathrm{pT}$ tracks for alignment
This is where we are 


\section{Cosmic 2008 and 2009}

- By the end of June 2009,

0.75M ID tracks (at least 1 pixel hit in each) are recorded
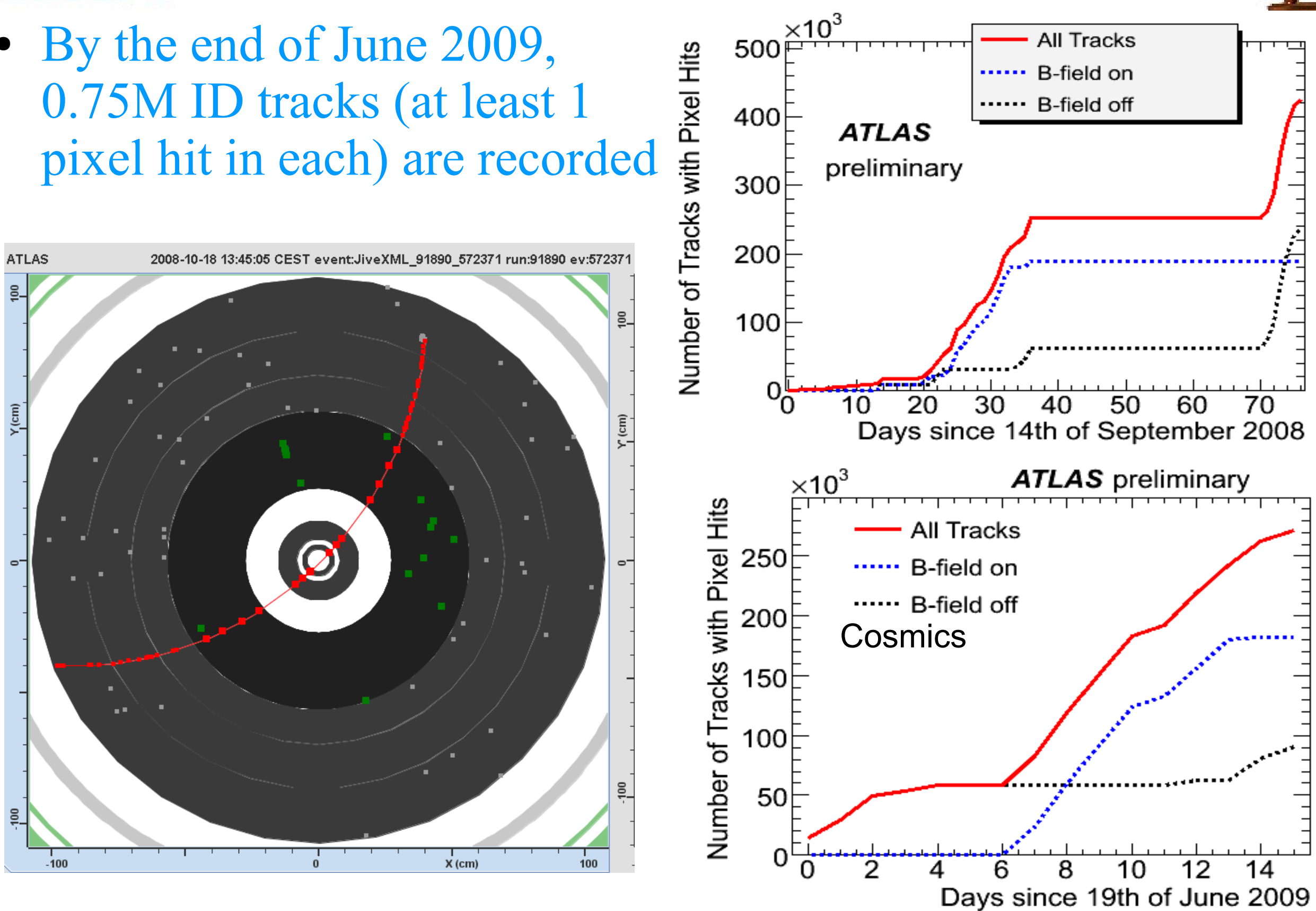


\section{Basic Validation: Cosmic}

- Use upper-lower half tracks to measure track parameter resolution

\begin{tabular}{lcc}
\hline \hline Parameter & \multicolumn{2}{c}{ Asymptotic resolution } \\
Track $\mathrm{p}_{\mathrm{T}}>30 \mathrm{GeV}$ & Cosmic-ray data 2008 & Monte Carlo \\
\hline$d_{0}[\mu \mathrm{m}]$ & $22.1 \pm 0.9$ & $14.3 \pm 0.2$ \\
$z_{0}[\mu \mathrm{m}]$ & $112 \pm 4$ & $101 \pm 1$ \\
$\phi_{0}[\mathrm{mrad}]$ & $0.147 \pm 0.006$ & $0.115 \pm 0.001$ \\
$\theta[\mathrm{mrad}]$ & $0.88 \pm 0.03$ & $0.794 \pm 0.006$ \\
$q / p\left[\mathrm{GeV}^{-1}\right]$ & $(4.83 \pm 0.16) \times 10^{-4}$ & $(3.28 \pm 0.03) \times 10^{-4}$ \\
\hline \hline
\end{tabular}
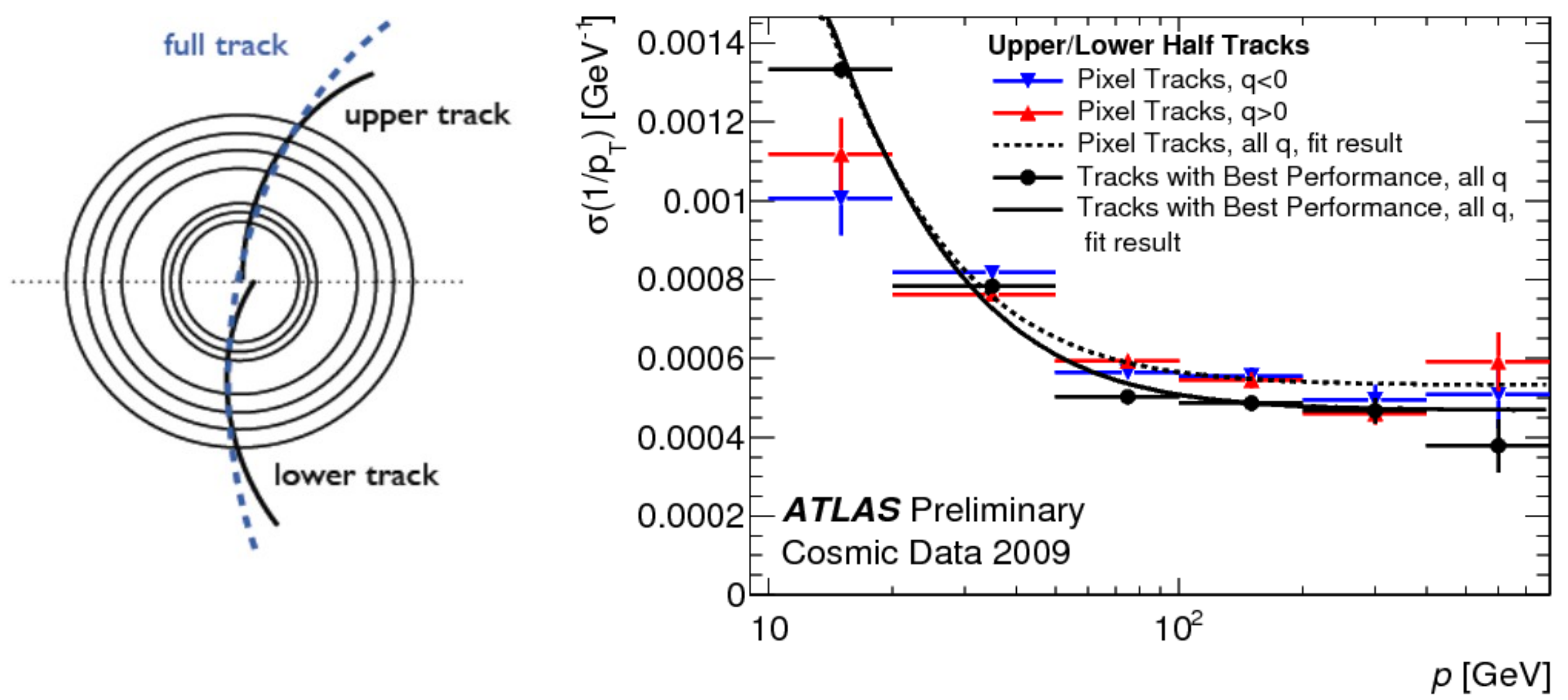


\section{Cosmic and Collision Data}

- Detector operates with high efficiency

- Accumulated high quality tracks (nSi $>=6$ ) for alignment

\begin{tabular}{|l|l|r|}
\hline SubDetector & Channels & Operation \\
\hline Pixels & $80 \mathrm{M}$ & $97.90 \%$ \\
\hline SCT Silicon Strips & $6.3 \mathrm{M}$ & $99.30 \%$ \\
\hline Transition Radiation Tracker & $350 \mathrm{~K}$ & $98.20 \%$ \\
\hline
\end{tabular}
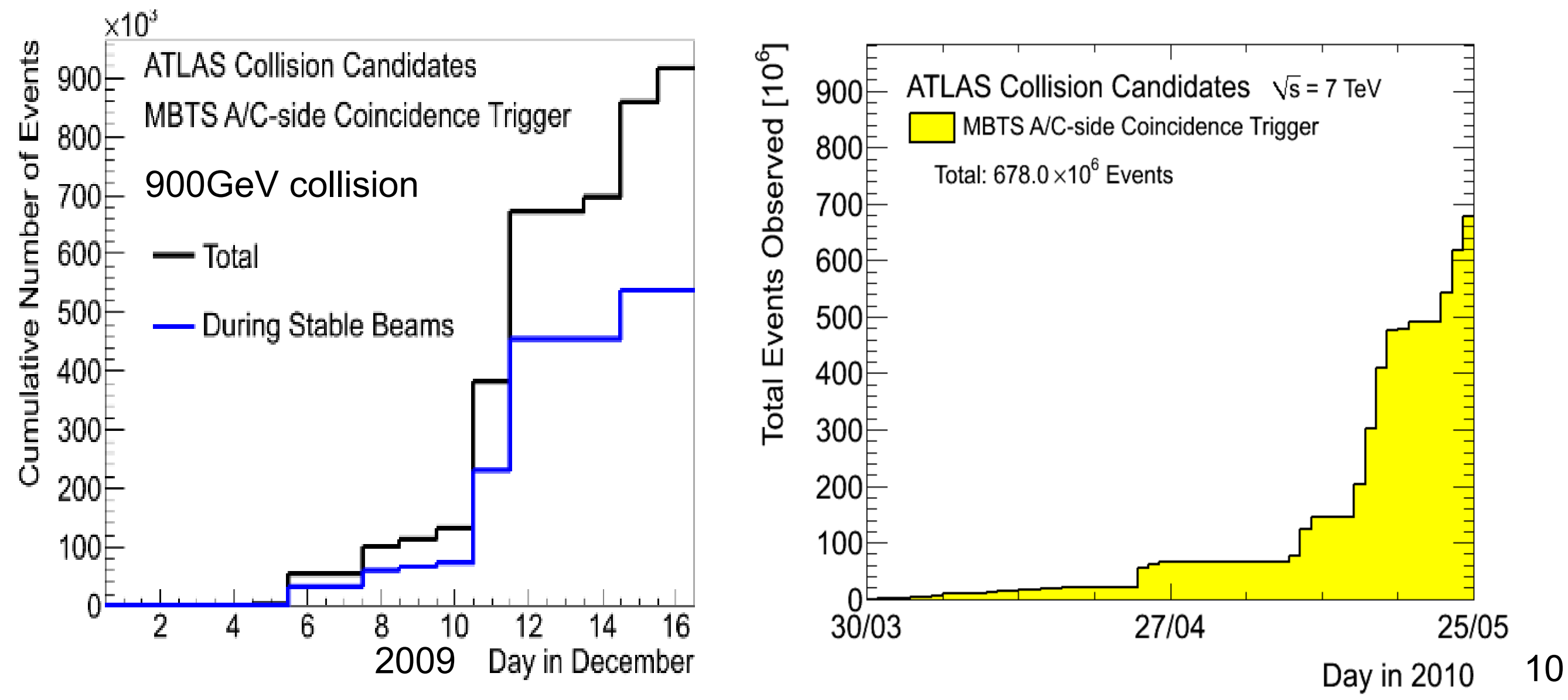


\section{Collision Data
Cirstion Alignment Using $900 \mathrm{GeV}$}

Dec 2009: Immediately after first $900 \mathrm{GeV}$ collisions, we have improved endcap alignment with $50 \mathrm{~K}$ tracks with $\mathrm{p}_{\mathrm{T}}>2 \mathrm{GeV}$

\begin{tabular}{|c|c|c|c|c|c|c|}
\hline \multirow{2}{*}{$\begin{array}{c}\text { narrow } \\
\text { Gaussian width }\end{array}$} & \multicolumn{2}{|c|}{ Pixel } & \multicolumn{2}{c|}{ SCT } & \multicolumn{2}{c|}{ TRT } \\
\hline Data $(\mu \mathrm{m})$ & 34 & 28 & 43 & 86 & 165 & 208 \\
\hline $\mathrm{MC}(\mu \mathrm{m})$ & 22 & 25 & 36 & 46 & - & - \\
\hline
\end{tabular}

\section{TRT:}

wider residual is due to older reconstruction release
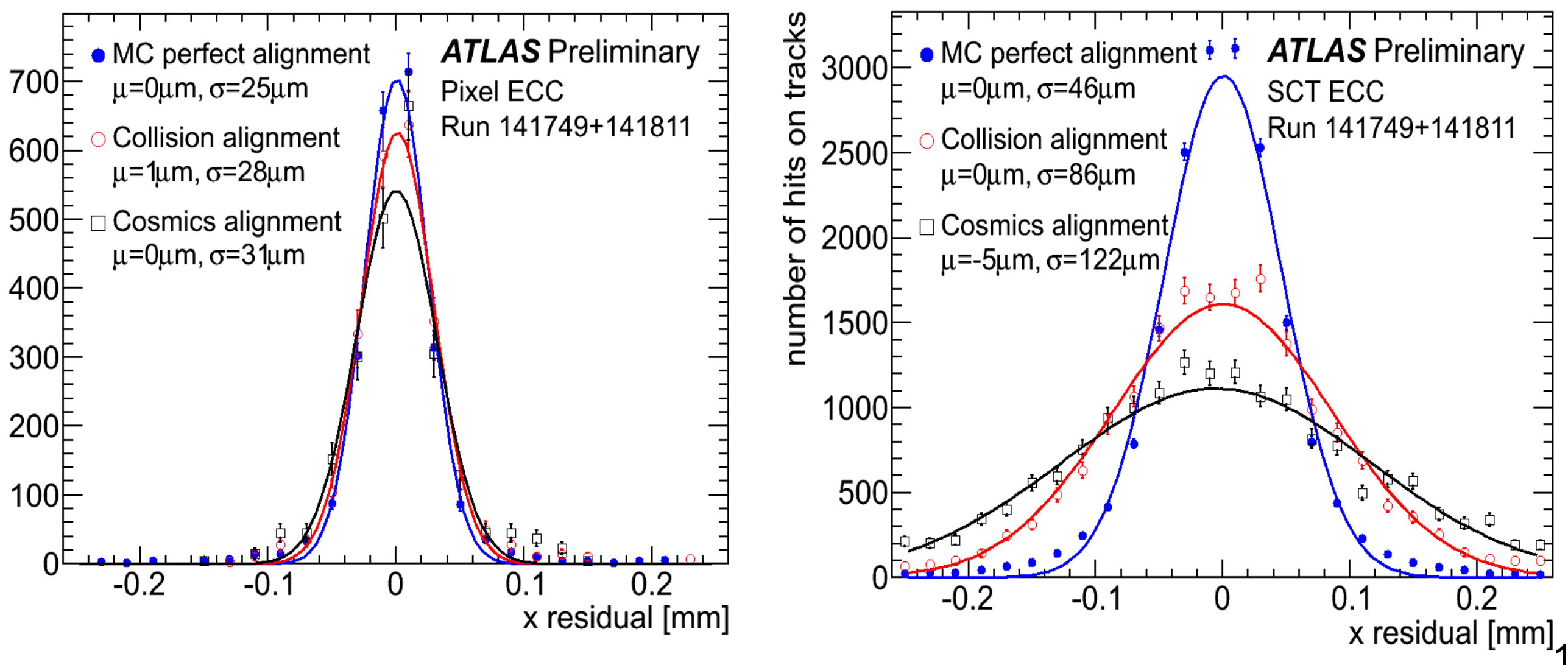


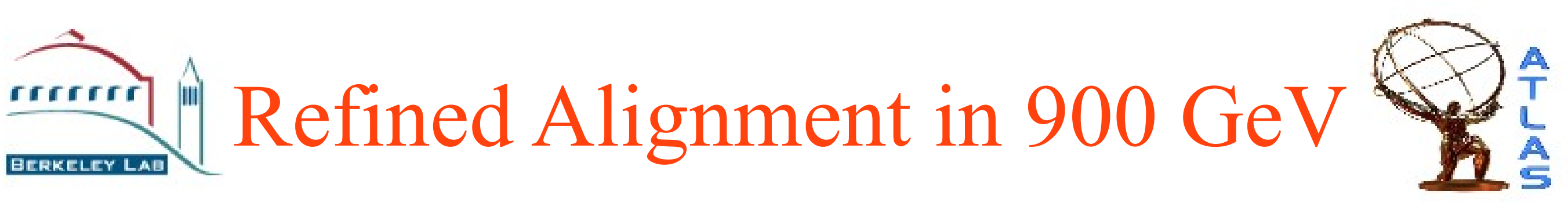

Feb 2010: Using full cosmic and $900 \mathrm{GeV}$ collision data and Robust + Global alignment to refine residual performance

\begin{tabular}{|c|c|c|c|c|c|c|}
\hline \multirow{2}{*}{ FWHM/2.35 } & \multicolumn{2}{|c|}{ Pixel } & \multicolumn{2}{c|}{ SCT } & \multicolumn{2}{c|}{ TRT } \\
\cline { 2 - 7 } & Barrel & EndCap & Barrel & EndCap & Barrel & EndCap \\
\hline Data $(\mu \mathrm{m})$ & 28 & 23 & 43 & 45 & 147 & 167 \\
\hline MC $(\mu \mathrm{m})$ & 23 & 20 & 38 & 40 & 145 & 143 \\
\hline
\end{tabular}
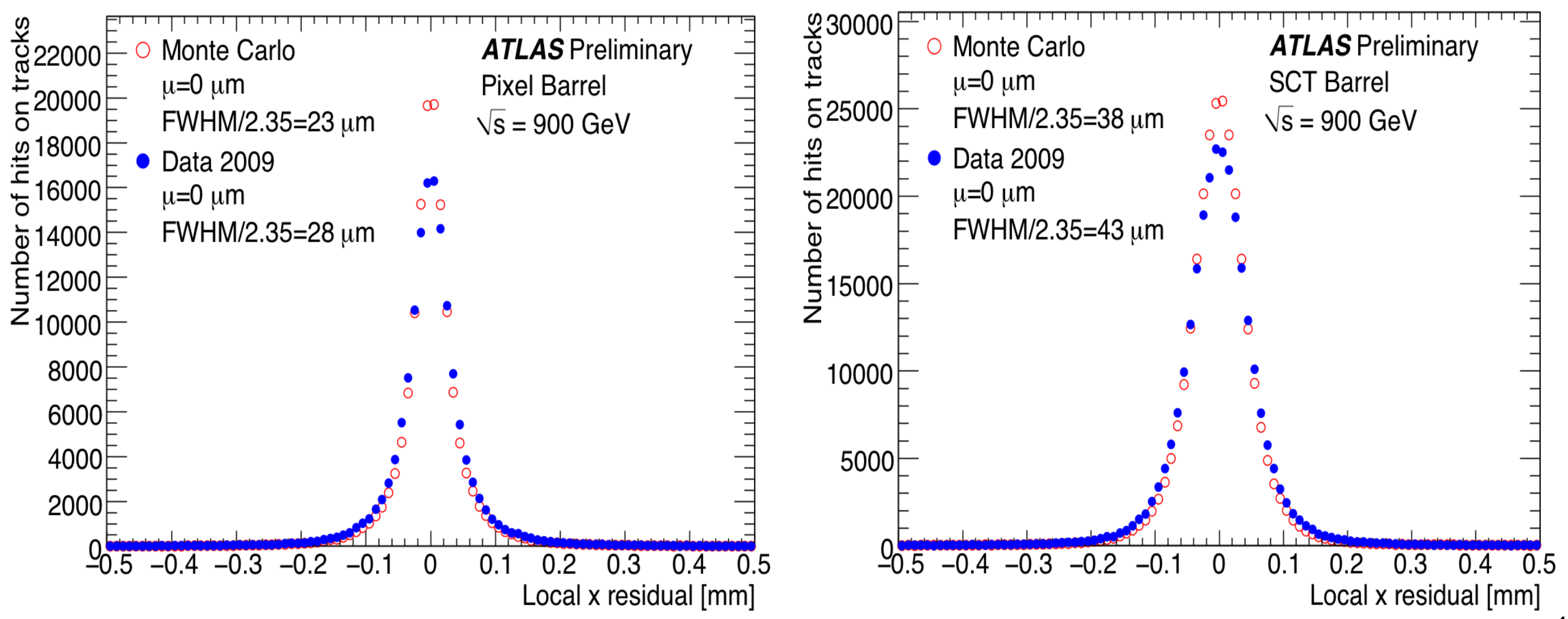


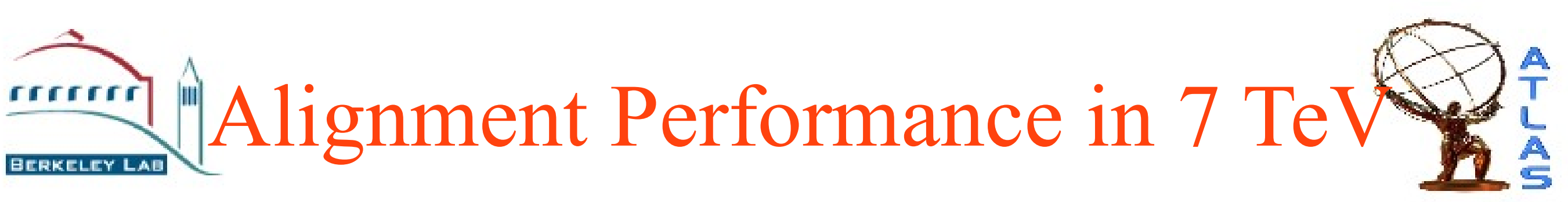

May 2010: Precision error and beam spot constraint are applied to pursue ideal resolution and eliminate systematic distortions

\begin{tabular}{|c|c|c|c|c|c|c|}
\hline & \multicolumn{2}{|c|}{ Pixel } & \multicolumn{2}{c|}{ SCT } & \multicolumn{2}{c|}{ TRT } \\
\cline { 2 - 7 } FWHM/2.35 & Barrel & EndCap & Barrel & EndCap & Barrel & EndCap \\
\hline Data $(\mu \mathrm{m})$ & 25 & 20 & 42 & 44 & 142 & 161 \\
\hline MC $(\mu \mathrm{m})$ & 18 & 19 & 34 & 38 & 143 & 135 \\
\hline
\end{tabular}

TRT:

Improved

digitization and

higher $\mathrm{p}_{\mathrm{T}}$ cut
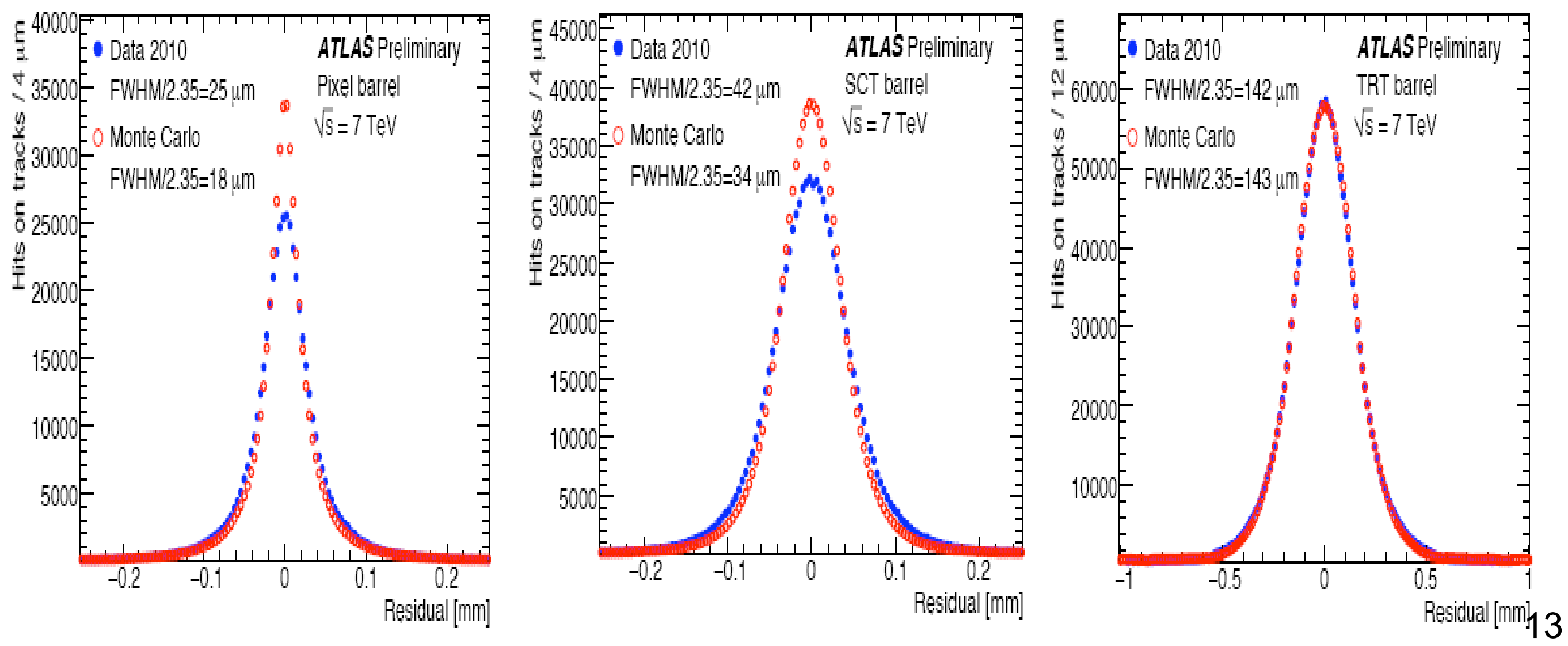


\section{Future Validation: Resonances}

BERKELEY LAB

- Resonance mass peak and width is sensitive to alignment effect

$\mathrm{K}_{\mathrm{s}}, \mathrm{J} / \Psi, \mathrm{Y}, \mathrm{Z}$

Curl (r vs $\Delta \varphi)$ Large 300um Small 20um

- Monte Carlo systematics studies with 9 simplified weak mode deformations:

$(\Delta \mathrm{r}, \Delta \varphi, \Delta \mathrm{z}) \times(\mathrm{r}, \varphi, \mathrm{z})$

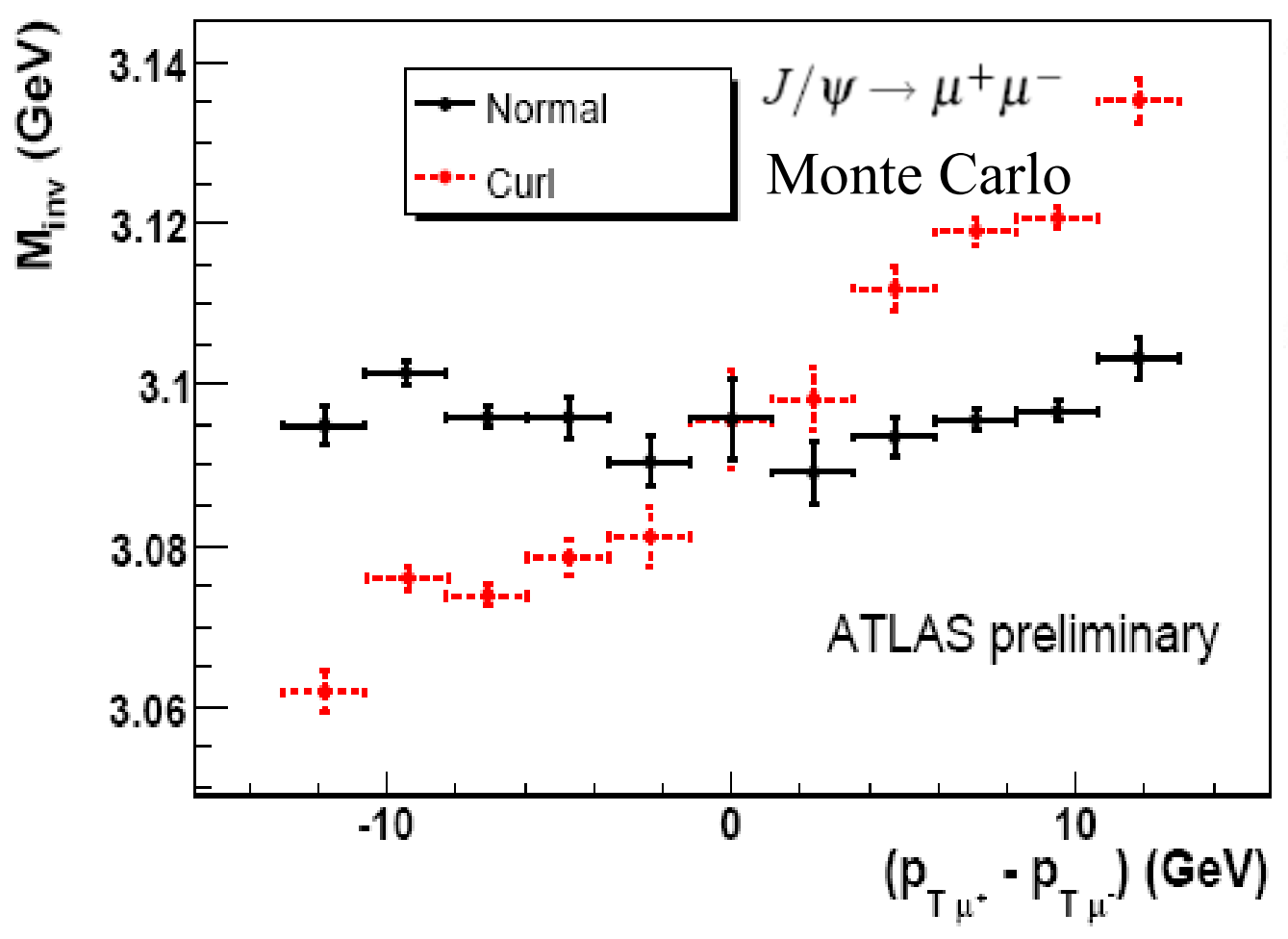

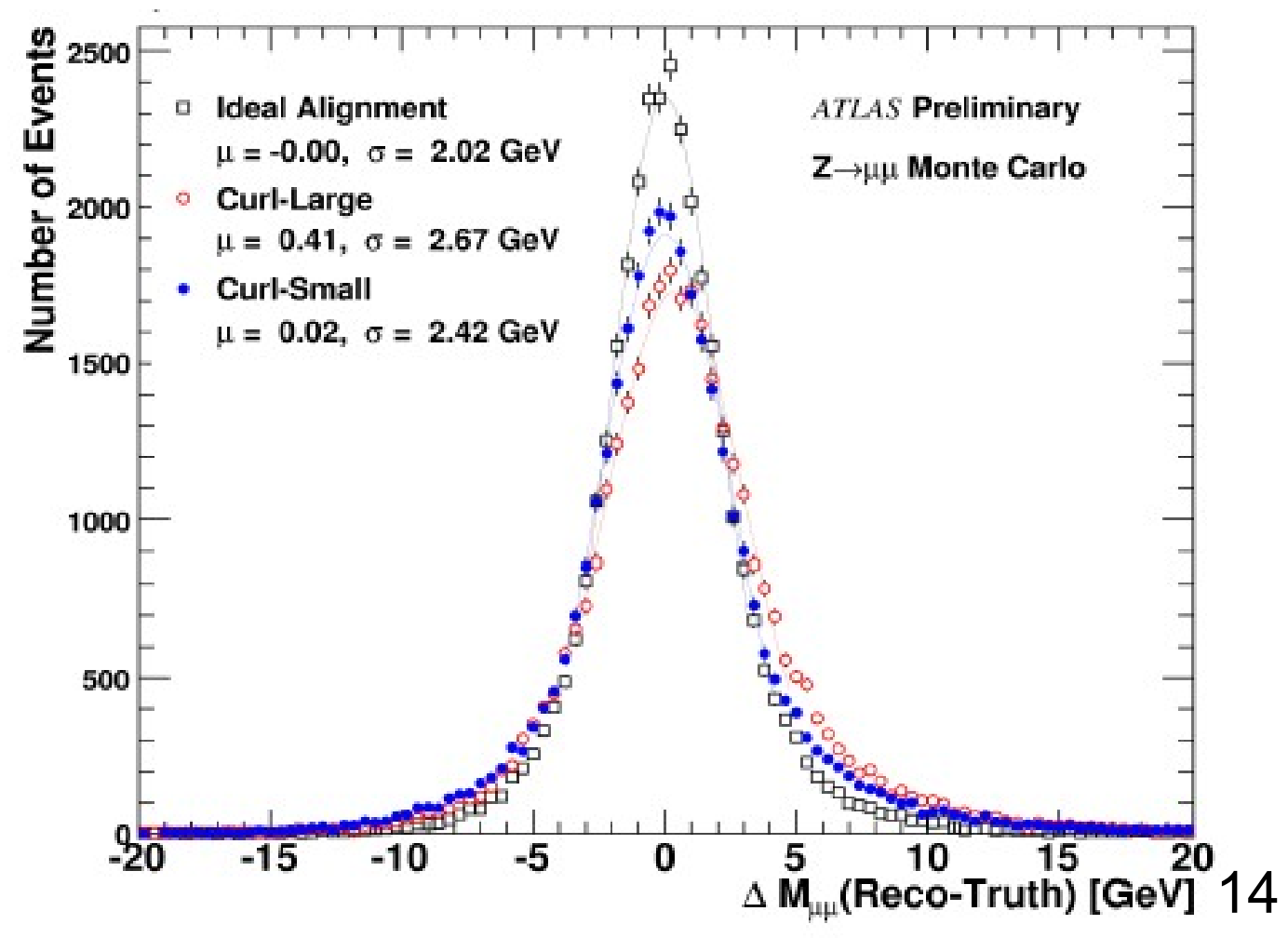




\section{Summary}

- ATLAS ID alignment applies state-of-the-art techniques: commissioned with cosmic and collision data

- Cosmic alignment is a great help and is served to reconstruct the first LHC alignment

- Alignment has moved from commission phase to precision phase. Looking forward more high $\mathrm{p}_{\mathrm{T}}$ tracks

- Restructured alignment code to integrate alignment algorithms and combined Si/TRT alignment

- Ready for higher luminosity data-taking and physics discovery in 2010 
